\title{
Categories of Modality and Their Use in Modern Biological Conceptions
}

\author{
Vitaly Ivlev \\ Moscow State Technical University named after N.E. \\ Bauman \\ (National Research University) \\ Moscow, Russia \\ E-mail: vitalijivlev@yandex.ru \\ Vladimir Inozemtsev \\ Moscow State Technical University named after N.E. \\ Bauman \\ (National Research University) \\ Moscow, Russia \\ E-mail: inozem_63@mail.ru
}

\author{
Marina Ivleva \\ Moscow Institute of Psychoanalysis \\ Moscow, Russia \\ E-mail: marinanonna@yandex.ru
}

\begin{abstract}
The paper discusses the validity of the use of the categories of modality (necessity, randomness and possibility) and clarifies the content of these categories in biology. For this purpose, the article, firstly, explores the first in the history of philosophy understanding of the categories of modality by Democritus, including by means of modern logic. Second, it identifies the main use of the categories studied in modern biology.
\end{abstract}

Keywords-categories; biological; necessity; randomness; possibility; modality; logic; methodology; scientific knowledge

\section{INTRODUCTION}

In modern philosophy considerable attention is paid to the study of the philosophical foundations of science. The composition of these foundations includes an ontological subsystem which is presented by a net of philosophical categories serving as a template for understanding and learning about the analyzed objects of special scientific pictures of the world (physical, biological, etc.). One of the most important of this class categories are categories of modality (necessity, randomness and possibility), which are used in philosophy and science since antiquity. These categories are widely used in modern biological concepts, being included in the philosophical foundations of biology. These foundations are a set of philosophical ideas and principles justifying the notions of the biological picture of the world, as well as the ideals and norms of biology and their coordination with the dominant ideology of the modern historical epoch. The paper discusses the question of the legality of using the categories of necessity, chance and possibility in the study of the philosophical foundations of modern biological concepts and of clarifying the content of these categories in biology.

(The study was funded by RSSF as a part of research project "Philosophical- methodological and natural science foundations of modern biological and environmental conceptions" project № 16-23-01004)

\author{
Mikhail Oseledchik \\ Department of Philosophy and Social Sciences \\ Moscow State University of Printing named after I. Fedorov \\ Moscow, Russia \\ E-mail: balu13@yandex.ru
}

The article addresses the following tasks:

- to explore the first in the history of philosophy understanding of the categories of modality (necessity, randomness and possibility) given by Democritus;

- to identify the main uses of the categories of modality in modern biological concepts;

- to formulate common generic and specific concepts of necessity, randomness and possibility.

\section{THE DOCTRINE OF NECESSITY, RANDOMNESS AND} POSSIBILITY IN THE PHILOSOPHY OF DEMOCRITUS

Appeal to the decision of the first of these problems is due to the fact that the first documented attempt in the history of philosophy to explore the understanding of the categories of necessity, randomness and possibility is taken in the philosophy of Democritus. In addition, such understanding of these categories (in the sense of Democritus' understanding) arises with the first attempts to formulate them before coming across with the relevant philosophical studies.

According to the testimony of ancient writers, Democritus had written 70 works. However, none of these products were preserved. The views of Democritus can be judged only thanks to the testimony of ancient authors. This evidence began to be collected by philosophers in the 19th century. So, in 1860, F. Mullah published nearly 300 pieces of evidence of the views of Democritus. In 1970, there were already published over 800 of them [Lurie]. Philosophical views of Democritus were studied in Russian literature by such scholars as A.O. Makovelsky, S.Y. Lurie, V.P. Goran, N.I. Styazhkin, V.S. Meskov and others. To a lesser extent are studied the logical ideas of Democritus. His doctrine of 
the categories of necessity and randomness also requires further study.

In the study of Democritus' views on understanding the categories of necessity, randomness and possibility are the most discussible in the first place. By the critical argument in favor of the fact that Democritus did not recognize the chance, many authors consider the statement about "an idol of randomness" which belongs to Democritus. Here, it is translated by A.O. Makovelsky "People forged the Idol of randomness to use it as an excuse to cover their own lack of common sense" [Lurie: Fragment 32]. This passage says that Democritus tells about the idol of randomness, indicating the randomness by "Tyche". This word for the ancient Greeks signified not just a chance, but a fate. Goran rightly argues that this statement criticizes the concept of Democritus fatal destiny [Goran, p. 30]. Thus, there is no basis for the claim that this or any other statements attributed to Democritus denied the randomness.

From the texts of witnesses and commentators of evidence we can conclude that Democritus calls "necessary" everything that is due to internal reasons and is inherent to things "by nature." Democritus calls random everything which is caused by external factors, and is not inherent to things "by nature." About this understanding it is said in the evidence 563 by Proclus [Lurie]. All the existing things Democritus divides into necessary which exist in all cases, and random, which do not exist in all cases. Random he subdivides into: (1) existing in the majority of cases (the possibility in the first sense); (2) (existing in the minority of cases (the possibility in the second sense); (3) existing in half of the cases - equal probability (possibility in the third sense). Thus, three types of chance are named by Democritus the possibilities.

In order to understand the views of Democritus on the categories of necessity and randomness, we should take into account his doctrine of atoms. Necessary for Democritus are the qualities which are due to the composition of atoms, and therefore, belonging to the bodies "by nature." These qualities belong to all bodies consisting of identical atoms, and they always belong to them. The qualities caused by the ways how atoms are connected are not owned by the bodies by "nature" as the bodies that affect each other and the arrangement of the atoms in the bodies because of the changes. These qualities are random because they belong not to all bodies and not always. Democritus does not speak about the following possibilities: "belonging to all subjects, but not in all cases", "belonging not to all subjects (some), but in all cases." Such cases cannot be true considering his doctrine.

\section{THE CATEGORIES OF NECESSITY, RANDOMNESS AND POSSIBILITIES IN BIOLOGY}

To identify on a content level the specifics of the categories of necessity, randomness and possibility, we should refer to the use of these categories in the modern biological concepts.

We single out the following basic cases of using these categories in biology:
- changes in the population as a result of genetic drift;

- signs of the body, necessary or incidental for its survival;

- mutations;

- genetic conditionality of the characteristics of the body.

First we consider case 1: the change of populations as a result of genetic drift.

In this case, the following concepts of randomness are used.

- Randomness 1: we call random the combinations of various allelic genes in $\phi$ reproductive cel. 1

- Randomness 2: individuals randomly choose their mating partners.

- Randomness 3: changes in the gene pool can randomly occur in small isolated populations (genetic drift).

Cases 1 and 2 are summarized in one concept: random is an event, if neither it nor its absence is determined by any external or internal factors. This is randomness $(1,2)$. This concept of randomness corresponds to the third notion of randomness according to Democritus, randomness as an equal probability.

Randomness 3 can be described and explained by the theory of random mass phenomena. Random mass phenomenon is a set of individual events. Random mass phenomena are described by generalizing induction through the selection of cases, excluding casual generalizations. The methodology of this induction includes a number of principles. Here are just the ones which can explain the phenomenon called genetic drift.

In logic and sociology, random mass phenomenon (the set of all the events that make up the phenomenon) is called the total combination. In our case, it is the population from which a part of individuals is not yet separated. Many subjects chosen for the study are called a sample, or a selected combination. It's a part of the initial population of individuals, which is separated from it and may develop into a new population.

The principles of subjects chosen from the general combination in the sample are as follows:

- The items from all subclasses of the total population should be selected. In this case for the study, we should select individuals from all subclasses with different genotypes of individuals forming these subclasses. As in the discussed case, the selection is made by nature in order to prevent the genetic drift. The separated part of the population should include the representatives of all genotypes;

- The number of items to be included in the sample of the educated subclasses of a total combination should be proportional to the value of these subclasses. For 
example, if according to the types of genotypes are formed three subclass, one of which includes half of all individuals and two other $1 / 4$, then the sample should have half of the animals of the first genotype, and the second half of the sample should include the representatives of the other two genotypes in equal amounts. If this principle "is not followed by the nature," a genetic drift can happen;

- It is necessary to take the optimal number of objects for the research. A situation, for example, may happen: we examine 100 items. In compliance with all other principles we obtain a certain result, we increase the number of studied objects to 500 . The result changes; we increase them to 600 . The result changes again, and with further increase in the number of studied objects, the changes in the result are not observed.

In a situation called genetic drift, nature itself "breaks these principles" (at least one of them). We mean that it actually happens so that a very small part of the population separates. In the separated part of the population, there are not the representatives of all genotypes, or the representatives of genotypes are presented not in the same proportion in which they are contained in the general population. The result is a violation of the terms of the gene pool reproduction. Conditions are violated as a result of external effects in relation to the initial population. Thus, by incident 3 should be understood the violation of conditions of reproduction of the gene pool under the influence of external factors (under the influence of changes in the conditions of existence of the population), and in the generalized form, incident 3 is something that occurs under the influence of external factor on the analyzed object (on the individual, the subject, system, etc.).

Genetic drift is a random phenomenon. If the above conditions and principles are met, the genetic drift does not occur. The law of the constancy of the gene pool of a population over a number of generations is happening - the law of Hardy-Weinberg. It should be noted that it takes place in a large population, when there is no mutation. Then, Mendel's second law takes play - law of independent distribution. There is no sense to speak about the compliance of the above methodological principles, except for the principle based on the law of large numbers because the statement refers not to the sample but to the general population. Hardy-Weinberg law acts in the case of separation of a part of individuals from the general population if all of these conditions and guidelines are followed. It states the need for constancy of the gene pool of a population. The nature of this concept is the same as the concept of necessity by Democritus: "it occurs in all cases and always". You can talk about this concept of necessity as a concept of what is conditioned by a system essence. The phenomenon is the constancy of the gene pool for generations. The essence (in this case) is what this constancy can be determined by, such as the above conditions and principles, which are treated in this situation as the properties of the system.
Thus, incident 3 is the one conditioned by the existence of the external environment of the system; necessity 1 is the one conditioned by the essence of the system. We can consider these categories as paired. Randomness as the equal probability or as indeterminacy of an event or lack of it is incident $(1,2)$.

We turn then to case 2: features of the organism, necessary or incidental to its survival. Within biological literature, they distinguish between necessary to the survival of the organism (adaptive) and random to the survival (nonadaptive) features. The latter are sometimes referred to as "useless." The necessary ones are those without which the organisms can not survive in these conditions, as well as those that provide benefits for organisms to survive in these conditions. The random ones are those that do not give any advantage to organisms to survive in these conditions.

The idea of inheritance needed characteristics for the survival of an organism and of loss of random characteristics for the the survival is the the core of the theory of natural selection by Darwin and Wallace. Other provisions of this theory are:

- animals and plants have the feature of variability;

- the organisms of a certain species have more births than they can find their food, so some organisms die;

- as there are born more organisms are born than the ones that can survive, among them there is a struggle for food and habitat;

- the fittest organisms survive.

We come to the following understanding of necessity, randomness and impossibility for survival.

The characteristics are necessary for the body if the survival of organisms that have this feature is determined by the conditions of their habitat. In defining the organisms, it is advisable to bear in mind the population, as in biology by a evolutionary unit they understand not a single individual but a population. In addition, the system, concerning which the issue of necessity or randomness is being solved, is the population together with the environment. Therefore, preserving required for the survival features for a number of generations is determined by the internal reasons of this system. These internal reasons (habitat) are the essence of the system. Vital for the survival of organisms is a feature, saving (but not the appearance of) which is determined by the inner essence of the system (population, considered together with the environment). We call this a necessity not by origin or the necessity 2 .

The feature is not possible (not by origin) if the system (population, together with the environment) is determined by the death of organisms with this feature. We call this impossibility 2 .

The feature is random (not by origin) if the system does not determine either its retention or its loss. We call this the randomness $(2,2)$.

The next is case 3: Mutations. 
One of the factors in the emergence of new genotypes and changes in the gene pool is a mutation process. In this case, the following concepts of necessity and randomness are used: necessity and randomness. Necessary to circumstances are the mutations caused by artificial means as a result of deliberate action on the chromosomes and genes. That is, the necessity in the circumstances can be referred to as the phenomenon, the existence or occurrence of which is determined by external circumstances. Randomness in the circumstances is when the mutations occur due to natural external causes, but it is not in all individuals. It's within the minority of them, and conditionality is not deterministic. Spontaneous incident is the mutations that occur sometimes for no apparent reason and only in individuals.

Finally, we turn to case 4: genetic conditionality of the characteristics of the organism. In the study of this conditionality of features the following concepts of necessity, randomness and possibility are used. Necessity is the unequivocal conditionality of the characteristic by the genetic code of an organism. Randomness is the ambiguous conditionality of the feature by the specificity of genetic material. Possible are the features inexplicitly determined by genetic abnormalities. There are used the possibilities 1, 2, and 3 in the sense of Democritus. Most often, the possibilities are expressed by numbers greater than 0 and less than 1 .

\section{THE TYPES OF NECESSITY, RANDOMNESS AND POSSIBILITY}

Based on the foregoing material, we can formulate generic and specific concepts of necessity, randomness and possibility.

First, we introduce the most common generic notion of necessity. The necessity is what is clearly determined by internal factors of the things, systems, etc. or external circumstances of their existence. The used by us concept of explicit determination can be explained by examples. For example, the electrical conductivity of metals is determined uniquely by the presence in them of free electrons, and some diseases are not uniquely determined by gene or chromosome abnormalities. That is, with these abnormalities depending on certain circumstances, the disease may occur or may not occur. Non-explicit determination exists objectively. It takes place not only in biology but also in other sciences. With explicit determination, the relevant reason is a sufficient condition for the occurrence of a specific consequence. With non-explicit determination, the cause is a sufficient condition for the emergence of one of a few specific results but which of them it is actually impossible to identify.

Synonymous to the category of necessity when applied to future events is the word inevitability. Treating the necessity as inevitability corresponds to both everyday and scientific use of the concept of necessity.

From the common generic concept of necessity, we move to the specific concepts of necessity. All of the earlier concepts of necessity (necessity in the sense of Democritus and understanding the necessity in modern biology) are specific in relation to this common generic notion of necessity.

We formulate the following specific concepts of necessity.

Classical (essential) necessity is what is strictly determined by the essence of things, systems, etc. An example of the essence can serve a genetic code of an organism. For example, at least in some cases, knowledge of the body's genetic code does not require deeper search of body's essence.

Functional necessity is a feature that is necessary if the conditions for the existence of its bearer explicitly determine the performance of certain functions by this feature bearer. An example of this concept is the notion of necessity not by origin, which is used in biology. Essential to the survival of organisms feature is the one the preservation of which is determined by the inner essence of the system (population) analyzed together with the environment.

The necessity by the circumstances is the phenomenon of the existence or occurrence of which is explicitly determined by external circumstances. Examples of such necessity are the mutations caused artificially by deliberate action on the chromosomes and genes. This concept can be applied to social phenomena.

Further, we define the most common generic concept of randomness. Randomness is what is not determined either by internal factors of the things, systems and etc. or external circumstances of their existence. The main specific notions of randomness can be formulated as follows. Classical randomness is a phenomenon that is non-explicitly determined by the essence of an object, system.

Functional randomness is a feature that's random if the conditions of existence of its bearer is non-explicitly determined or the execution of certain functions by a feature bearer that is not determined. An example of such randomness can be the described in the preceding chapter the randomness not by origin.

Randomness by the circumstances is the phenomenon, the existence or occurrence of which is not clearly determined by external circumstances.

Finally, we introduce the most common generic term possibility. The possibility is something the absence of which is not clearly determined by either internal factors or external circumstances.

Based on this understanding of possibility, all necessary is possible. The types of this common generic notion of possibility can be the possibilities, quantitatively characterized by the theory of probability, i.e. by taking as a measure of probability of numbers (rational) greater than zero and less than one. The particular cases of possibilities in this approach are the possibilities, which are a generalization of possibilities by Democritus:

B1 - feature inherent in most objects in most cases; 
B2 - feature inherent in the majority of objects in a minority of cases;

B3 - feature inherent in a minority of objects in most cases;

B4 - feature inherent in half of objects in half of cases;

B5 - feature inherent in a minority of objects in a minority of cases.

Philosophical categories belong to the worldview part of science (its philosophical foundations). On the basis of the categories are developed methodological principles as well as methods and techniques of cognitive and practical activities. They serve as a guide in the cognitive and practical activity and are responsible for a certain view of reality. The philosophical view of reality contributes to its more successful learning. The system of categories is like a net, by imposing which on reality we create the benchmarks for understanding it. Knowledge of philosophical categories contributes to the most fruitful dialogue between the representatives of various specific sciences as well as the representatives of the various branches of the same science. The system of philosophical categories is the basis of the language of interscientific and intrascientific communication.

Knowledge of the types of necessity, randomness and possibility facilitates the study of specific phenomena as it sets to searching for specific conditionalities in a particular section of a particular science. In those cases, when there are detected events which do not fall under the described types of necessity, randomness and possibility, the task of science is to develop new concepts. Then, these new concepts will be used by philosophy to summarize the previously established concepts, and the results of generalizations will be used by specific sciences.

\section{CONCLUSION}

The article solved the tasks formulated in the beginning of the work:

- is described the understanding of the categories of modality (necessity, randomness and possibility) by Democritus;

- are identified the main uses of the categories of modality in modern biological conceptions;

- is carried out the generalization of concepts about different kinds of necessity, randomness and possibility and on this basis the corresponding philosophical categories are formulated.

However, in the process of this research there appeared new problems that require solutions.

- It is necessary to supplement the study of the use of the categories necessity, randomness and possibility in modern biological conceptions with the study of their use in environmental conceptions.
- To examine in more detail the development of the mentioned categories by modern philosophers and philosophers of the past.

- To investigate the opportunity not to use the categories of necessity, randomness and possibility in scientific cognition. If such an opportunity exists, it is necessary to show the advantage of using the discussed categories or to abandon their use in favor of another system of categories which is, in our opinion, unlikely. In addition, there should be carried out the work to clarify not only the categories of necessity, randomness and possibility, but also other categories (nature, determinism, and so on). It is possible that such work can result in the clarification of philosophical categories.

\section{REFERENCES}

[1] Voishvillo E.K. The concept as a form of thinking. Moscow, 1989.

[2] Goran V.P. Necessity and randomness in the philosophy of Democritus. Novosibirsk 1984

[3] Greene N. , Stout W. , Taylor D. Biology. V. 1-3. M., 1990.

[4] Ivleva M.L., Inozemtsev V.A., Ivlev V.Y., Udovik V.E. Information society and the formation of a new epistemological paradigm of modern science.- M. 2013.

[5] Lurie S.Y. Democritus. Texts. Translation. Research. L., 1970

[6] Makovelsky A.O. Ancient Greek atomists. Baku 1964

[7] Petrov Y.A. Methodological issues of applying and developing scientific concepts. M., 1980.

[8] Vogel F., Motulsky A. Human Genetics. V. 1-3. Moscow, 1989.

[9] Anderson A., Belnap N. Entailment: The Logic of Relevance and Necessity, vol. 1 (1975), vol. 2 (1992). Princeton: Princeton University Press.

[10] Benthem J.F. van. Modal Logic and Classical Logic. Naples: Bibliopolis, 1983.

[11] Blackburn P., Wolter F. Handbook of Modal Logic. Amsterdam Elsevier. 2007.

[12] Bull R., Segerberg K. Basic Modal Logic // D. Gabbay and F. Guenthner (eds.), Handbook of Philosophical Logic. II.1. Dordrecht: D. Reidel, 1984. 1-88.

[13] Carnielli W., Pizzi C. Modalities and Multimodalities. Heidelberg Springer-Verlag, 2008.

[14] Gabbay D. Investigations in Modal and Tense Logics. Dordrecht: D. Reidel, 1976

[15] Hughes G., Cresswell M. An Introduction to Modal Logic. London: Methuen, 1968.

[16] Kripke S. Semantical Considerations on Modal Logic // Acta Philosophica Fennica, 1963. 